\title{
13 Loop quantum gravity and exploding black holes
}

\author{
Simone Speziale \\ Centre de Physique Théorique, UMR7332, CNRS, Université d'Aix Marseille \\ \& Université de Toulon, 13288 Marseille, France
}

\section{Abstract}

Black holes are one of the most fascinating objects populating the universe. According to classical general relativity, they are eternal and can only grow bigger in time. Hawking was the first to show that taking quantum effects into account can change the picture, leading to a slow evaporation of black holes by quantum thermal radiation. This theoretical phenomenon, yet to be observed, raises crucial conceptual and technical questions aimed at any fundamental theory of quantum gravity. Among these, the famous information-loss paradox. I will present two different hypothesis to address these questions: a more conservative one, based on modified Einstein's equations and non-singular black holes; and a more speculative one, based on loop quantum gravity and exploding black holes, which may also lead to interesting observational consequences. 


\section{Résumé}

\section{Gravité quantique à boucle et trous noirs explosifs}

Les trous noirs figurent parmi les objets les plus fascinants peuplant notre univers. Selon la relativité générale dans sa version classique, ils sont éternels et ne peuvent que croître avec le temps. Hawking a été le premier à montrer que la prise en compte des effets quantiques peut changer la donne, en conduisant à une lente évaporation par radiation thermique quantique. Ce phénomène théorique, qui a encore à être observé, soulève des questions cruciales, conceptuelles et techniques, visant toute théorie fondamentale de gravitation quantique. Parmi ces questions, le fameux paradoxe de la perte d'information. Ce chapitre présente deux hypothèses différentes pour tenter d'y répondre : une première hypothèse, la plus conservatrice, est basée sur une modification des équations d'Einstein conduisant à des trous noirs avec absence de singularité ; une seconde, plus spéculative, est basée sur la gravité quantique à boucle et des trous noirs explosifs, hypothèse qui peut également conduire à d'intéressantes conséquences potentiellement observables.

\section{Introduction}

Right on time for the hundredth birthday of Einstein's theory of general relativity, gravitational waves have been observed by the two American laser interferometers led by the LIGO consortium. This extraordinary result has perfectly confirmed once more Einstein's predictions. The difficulty of the observation, the reason why it took so long, lies in the extreme weakness of gravity compared to the other forces: even though the whole Earth is pulling the chalk I hold towards the ground, it takes me a small effort to beat this attraction, using the chemical energy of my muscles, ultimately of electromagnetic origin. For the same weakness, another extraordinary prediction of GR, that is the slowing down of time with the increase of the gravitational field had to wait until the seventies for experimental confirmation, with the building of the first GPS satellites. Around the same time came also the first indirect observation of gravitational waves: an orbital pair of neutron stars with a strong magnetic field was observed losing energy and slowing down at exactly the rate predicted by GR if that energy was carried away in the form of gravitational waves. Direct observation of those waves was not possible at the time, as we did not have an instrument sensitive enough. Now we have, thanks to LIGO, and the era of listening to the universe via gravitational waves has just begun, with the upcoming construction of more laser interferometers around the world.

Black holes also have been predicted using Einstein's theory long before their observation. As early as three months after the publication of the equations of general relativity, Schwarzschild found the simplest spherically symmetric and static solution. This solution describes the deformation of spacetime around a star or a planet, and introduces small but crucial corrections to Newton's description of gravity. In particular, it leads to a correction to the precession of the perihelium of Mercury and to the deflection of light, the two immediate confirmations of general relativity, and includes the slowing down of time, 


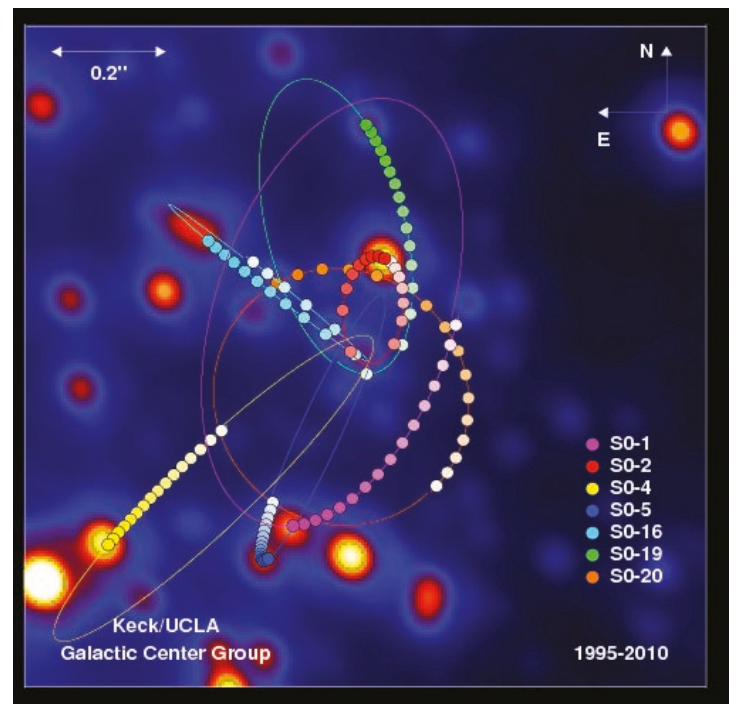

Figure 1

an effect called gravitational redshift. Remarkably, the gravitational redshift and associated slowing down of time can become increasingly large, the more the star is compact. If a star of mass $M$ could be compressed in a region of radius smaller than the critical value $r_{S}=2 G M / c^{2}$, the effect would become infinite. This infinite redshift implies also an infinite redshift of any propagating wave, so that nothing, not even light, can emerge from the region inside the surface of radius $r_{S}$, thereby named the event horizon: an outside observer has no access to events happening inside this surface.

Initially considered to be just a theoretical abstraction, the notion of such 'black holes' become experimental evidence with the observation of regions of spacetime sourcing strong gravitational field (observed through the effects on the surrounding matter) and being completely invisible. The first such observation dates back to 1964 and the $X$-ray source Cygnus $X I$, now understood to be caused by the matter falling into a black hole from a giant star orbiting around it [ $I]$. Since then we have discovered a large number of black holes, in mass ranges that go from a few solar masses, to monsters the equivalent of a hundred million Suns. We even have pictures of them! See for instance this famous image by the KECK telescope (figure I), showing the center of our galaxy: The highly elliptical orbits of a few stars have been reconstructed over a 15-year period, all turning around a dark object the mass of a million Suns.

Observational confirmation of black holes, even if restricted to the region outside the event horizon, stimulates questions and theoretical thoughts on what lies beyond: what truly happens at the event horizon? What happens inside? From the mathematical viewpoint, these questions can be answered using general relativity. The theory tells us for instance that an observer falling inside the black hole will cross the event horizon in a finite time 
and without experiencing any extremal conditions. Looking back at the direction he is coming from, he would see the evolution of its visible universe accelerate exponentially in front of his eyes, like in accelerated motion on a video. As he keeps falling in however, the gravitational attraction increases enormously in strength, until the tidal effects are so strong to destroy the observer spaceship and the observer himself: in Einstein's theory, the gravitational strength grows unboundedly as we approach the center of the solution, where it becomes infinite. This so-called curvature singularity at the centre of the black hole is a region where all infalling trajectories end: there is no escape from it, and everything that falls in the black hole is doomed to destruction. Is this really what happens inside a black hole? We will come back to this question below.

In any case, the strange interior region with its singularity lurking in the dark does not affect the physical application of general relativity to astrophysical phenomena, since the singularity is hidden by the event horizon and thus causally disconnected from our universe. Indeed, scientists like Hawking, Penrose and many others developing the classical theory of black holes and general relativity, conjectured that all such singularities are hidden by event horizons. This was called the Cosmic Censorship Conjecture, and it is supported by the mathematical evidence that gravitational collapses form event horizons around singularities. Furthermore, Hawking proved that an event horizon can never decrease in size, but only increase, as new matter falls in the black hole, thus proving that black holes are eternal in classical general relativity.

Shortly after proving the theorem, Hawking realized that quantum mechanical effects would violate one of its hypotheses, in particular, the positivity of energy. Think of instance of anti-particles, or of quantum tunnelling effects. Quantum mechanical effects could then lead to a black hole losing mass and shrinking. Now, including quantum mechanical effects in general relativity is a very delicate issue: the very foundation of quantum mechanics and quantum field theory, its extension to systems with infinitely many degrees of freedom, requires a fixed and flat Minkowski spacetime and its associated isometries, like in special relativity. On the contrary, in general relativity spacetime is dynamical, not fixed and lacks in general the isometries of Minkowski. We will come back to this below, but as an intermediate step, it is possible to consider quantum field theory on a fixed but curved spacetime, provided it is stationary. This set up is strongly limited because it ignores the back-reaction of matter on spacetime, thus the heart of Einstein's theory, but it can provide a simpler setting to test ideas and physical effects when the matter content has little energy content. In this approximate setup, Hawking was able to derive a stupendous conclusion: indeed, quantum effect would lead to energy leaking out of the black hole. The leaking takes the form of a thermal radiation of quantum particles at a temperature given by

$$
T_{\mathrm{H}}=\frac{\hbar}{8 \pi G M},
$$

where $M$ is the mass of the black hole.

Intuitively, this effect can be understood as a gravitational version of Schwinger's vacuum polarisation: the quantum vacuum of the field is teeming with particle-antiparticle 
virtual pairs, and if we apply a strong enough electric field say with a capacitor, the pairs can turn into real particles, separated from each other and attracted to the two opposite plates of the capacitor. The energy to excite real particles from the vacuum is provided by the capacitor. In the Hawking effect, it is the strength of the gravitational redshift near the event horizon that polarises the vacuum, and the energy to create real particles is subtracted to the black hole itself. What is surprising about Hawking's result is the perfectly thermal nature of the quantum radiation. A black hole then behaves exactly as a black body in thermodynamics. Hawking's result is just a first order approximation and it ignores the back reaction. Hence his theory does not tell us how the black hole changes when a quantum of radiation is emitted. But the thermodynamical behaviour of the black hole, suggested by many other properties discovered around the same time such as the famous area entropy law, suggests that we can use Stefan-Boltzmann law to estimate the energy loss rate. This gives

$$
\dot{M}=-\frac{\hbar c^{6}}{G^{2} M^{2}}
$$

If there are no important higher order or non-perturbative corrections appearing, the evaporation will make a black hole lose all its mass in a finite time: integrating (2), we get

$$
\tau_{\mathrm{H}} \sim \frac{G^{2}}{\hbar c^{6}} M^{3}
$$

Black holes may not be eternal after all!

Such a finite lifetime is by no means in contradiction with the observed existence of black holes, as for macroscopic black holes is much longer than the age of the universe itself. For instance, the Hawking lifetime of a solar mass black hole is $10^{67}$ years! Only for microscopic black holes this finite lifetime could be tested; however, although suggested by some theorists, microscopic black holes have never been observed. Furthermore, Hawking's radiation itself is not astrophysically observable: for a solar mass black hole, $T_{\mathrm{H}} \sim 10^{-8} \mathrm{~K}$ is smaller than the cosmic microwave background of $2.7 \mathrm{~K}$.' Even lacking direct testing, Hawking radiation has been rederived in a plentiful of different ways in the passing years, and very few doubt of the solidity of this theoretical result.

From a theoretical point of view, Hawking's radiation poses a number of extraordinary challenges that go hand to hand with the issue of the central singularity. In fact, if classically we could invoke the Cosmic Censorship Conjecture and assume that all singularities are hidden from the eye, and general relativity can be applied without having to deal with them, Hawking's result on the evaporating event horizon brings out the singularity again. To deal with the singularity, it may then be necessary to go beyond general relativity, beyond also the approximate scheme of quantum field theory on curved spacetimes used by Hawking, and deal with a proper quantum theory of gravity.

(I) Efforts of verifying Hawking's radiation rely on so called analogue systems [2]. 
Applying quantum mechanics to general relativistic systems requires thus going beyond the actual foundations of theoretical physics, and it is considered by many the holy grail of theoretical physics. This quest has fascinated the most brilliant minds for over 80 years now, and a large number of very creative proposals have been put forward. While none of these ideas has led to consistent experimental predictions that could be verified, this question has blossomed into a whole research field that regularly produces important advances in theoretical physics in general, as well as mathematics and foundations of quantum mechanics. In this field the most developed and most followed approach is certainly string theory. The trouble with string theory is that the very foundations of the theory require the existence of supersymmetry and extra-dimensions, and an ever increasing amount of experimental evidence has been building against these two ideas, based on designed experiments failing to see the observed effect. An orthogonal approach, which does not require extra-dimensions nor supersymmetry, but instead focuses on the dynamical nature of spacetime in general relativity, is offered by loop quantum gravity (LQG) [3]. This approach, pioneered by Abhay Ashtekar, Lee Smolin and Carlo Rovelli (whose 60th birthday will be celebrated this year with a conference in his honour in Marseille) will be discussed in more details below.

Summarizing, black holes and Hawking radiation provide a perfect testing ground for any candidate theory of quantum gravity. In particular, we can select three key questions that should be addressed:

The dynamical problem: Is Hawking's evaporation formula valid throughout, i.e. does the black hole evaporate completely?

The singularity problem: If it evaporates completely, what happens to the central singularity? Does it become exposed, does it vanish?

The information paradox: Hawking's radiation is perfectly thermal; if the singularity is absent, what happens to the information of the matter that fell in the black hole?

The situation with the curvature singularities of general relativity is reminiscent of an analogous problem faced by theoreticians in the early 20th century, namely the classical instability of atoms. According to Maxwell's theory of electromagnetism, the electrons spinning around the charged nuclei should have emitted radiation, thus losing angular momentum and inevitably falling into the nucleus. In other words, the classical potential had a singularity at $r=0$. The solution to this problem came from the inclusion of quantum effects: the uncertainty principle introduces an effective quantum pressure preventing the electron from being localised in the centre with zero momentum. Even in the absence of a complete theory of quantum gravity, many researchers conjecture that something similar will happen also in the gravitational case. Planck-scale quantum gravity effects will modify Einstein's equations, introducing some sort of effective pressure halting the gravitational collapse. The singularity theorems of Hawking and Penrose are violated simply by the modification of Einstein's equations.

There is a vast literature of ideas and results concerning these questions and similar variations. In the following, I will briefly present two different hypothesis on which I have 
worked: non-singular black holes [9, 10] and exploding black holes [II]. The two hypotheses share the same fundamental idea, that the fundamental theory of gravity resolves the central singularity, but differ in the detailed realisation and physical consequences. In the case of non-singular black holes, the quantum pressure stops the collapse to a somewhat steady state, still looking like a black hole from the outside, but non singular at its centre. Hawking radiation then comes into play, slowly evaporating away the black hole, finally exposing the inner core of the collapse, thus restoring the information lost in the collapse. In the case of exploding black holes, the quantum pressure is so strong that it leads to a 'bounce' of the gravitational collapse, turning the in-falling matter into an outgoing, exploding shell. The relevant quantum effect is a tunnelling process, and Hawking radiation is treated as a sub-leading, dissipative effect.

\section{Non-singular black holes}

Many tentative theories of quantum gravity predict modifications to the Einstein's equations. The modifications can be organised in a power series in the quantum gravity parameter $L$ (ideally proportional to the Planck length $\mathrm{P}_{\mathrm{P}}=\sqrt{\mathrm{hG} / \mathrm{c}^{3}}$ ),

$$
R_{\mu \nu}-\frac{1}{2} g_{\mu \nu} R=\frac{16 \pi G}{c^{4}}\left[T_{\mu \nu}+L \ldots+L^{2} \ldots+\ldots\right] .
$$

A typical case study for instance is when the extra terms in the ellipses contain higher powers of the Riemann tensor $R_{\mu \nu \rho \sigma}$. The modification of the theory invalidates the singularity theorems, and solutions with non-singular black hole metrics can now be found. To be interesting, such solutions should satisfy three criteria:

(i) look like a black hole from outside the event horizon, so to be compatible with general relativity;

(ii) be regular everywhere, in particular have no central singularity;

(iii) have all curvature invariants everywhere sub-Planckian in magnitude:

$$
R<\ell_{\mathrm{P}}^{-2}, \quad R_{\mu \nu \rho \sigma} R^{\mu \nu \rho \sigma}<\ell_{\mathrm{P}}^{-4}, \text { and so on. }
$$

The third criterium is needed to justify the use of a classical, smooth metric to describe quantum gravity effects. The point here is that nothing guarantees that a metric description of quantum gravity is possible above the Planck scale. String theory and loop quantum gravity for instance, argue that this is not possible, and a completely different fabric of spacetime emerges at those scales. Therefore the smooth non-singular black hole metric makes sense only to describe regions of sub-Planckian curvature, albeit extremely high. These conditions by themselves turn out to impose important restrictions on the allowed metrics, so that it becomes interesting to study non-singular black hole metrics satisfying $(i-i i i)$ in their own right, regardless of the details of the underlying fundamental theory. 

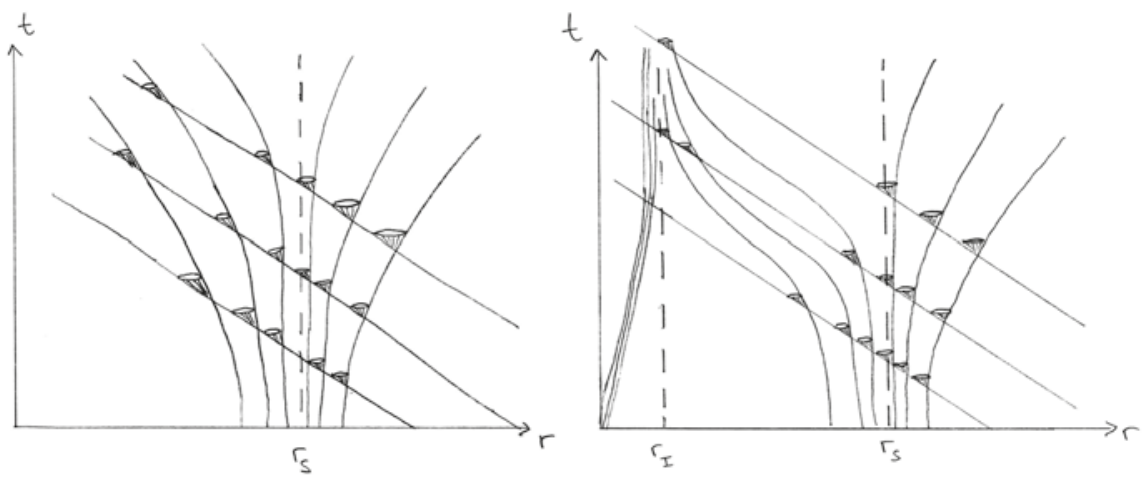

Figure 2

In particular, it is possible to prove that a spherically symmetric non-singular metric that looks like a black hole from far away must have a second horizon inside the event horizon, thus making the central location a time-like region. The new inner horizon has the opposite properties of the event horizon: it is an infinite-blueshift surface, and an accumulation region for geodesics, see figure 2 . While this is a rather generic property of non-singular black holes, the details depend of course on the precise metric used. The case study most developed in the literature was introduced some ten years ago by Hayward [4] (see also [5]). Its light cone structure is shown in the figure 2 (right panel), in comparison with the one of Schwarzschild (left panel): We see the presence of a second horizon, acting as an accumulation surface for the outgoing null rays. The central region of the metric has no singularity, but a finite value of the curvature inversely proportional to $L$ in (4), so that if we switch off the deviations from Einstein's equations, namely we take the limit $L \mapsto 0$, we recover the central singularity. With a non-singular central region, the physical situation of this new black hole is similar to what happens at the centre of a star, where one observer could be static in free fall (provided he doesn't burn!).

What happens if we try to address the puzzles of evaporation in this context? We can assume that the mass evaporates in time according to Hawking's law (2), and see what happens to the resulting spacetime. Since the inner horizon acts as an accumulation surface, all the matter that falls in to form the black hole accumulates at the inner horizon. As the outer, event horizon slowly evaporates for Hawking radiation, matter continues to accumulate. Assuming that the Hawking evaporation goes through all the way, at some point the even horizon will rejoin the inner horizon, and the two will merge together and disappear, leaving the central region exposed. As there is no more a singularity in the centre, this evolution is perfectly compatible with the Cosmic Censorship Conjecture. Furthermore, it answers the information-loss paradox quite simply: the information on the matter fallen in is by no means lost, and becomes available once the event horizon has disappeared. ${ }^{2}$

(2) Technically, what happens is that the thermal Hawking radiation emitted at early times is purified by the outcoming radiation after the complete evaporation of the event horizon. 
This qualitative picture is compelling, it provides a simple and elegant answer to both the singularity resolution and the information paradox. The difficulty is to find a consistent quantum theory of gravity confirming this picture and describing its details. In particular, properly taking into account the back-reaction of Hawking radiation on Hayward's metric, which means also verifying the assumptions that the black hole evaporates completely, requires solving the equations (4) with the specific modification included, and with $T_{\mu v}$ on the right hand side computed as expectation value of the quantum field on the Hayward metric. This computation is extremely difficult and we have no explicit answer yet. Nonetheless, preliminary calculations expose the following potential problem: because of the accumulation surface, all the information is released at once, when the event horizon completes its evaporation. This release would be associated with a huge burst of energy much larger than the mass of the black hole itself, and thus incompatible with standard energy conservation. It is possible that a special form of the extra terms in (4) can be found, such that the scenario above is realized resolving this problem with energy conservation, but this we do not know yet.

In conclusions, the hypothesis of non-singular black holes is quite appealing for its simple resolution of the information loss paradox, but needs to be still improved before it can be given substantial theoretical evidence.

\section{Quantum tunnelling and exploding black holes}

Among the alternatives, a more speculative idea has been recently put forward by Carlo Rovelli and his research group $[6-8, I I]$. The basic idea is that when the energy density of the collapsing matter reaches the Planckian density (i.e. $\rho_{P}=c^{5} / \hbar G^{2} \sim 10^{96} \mathrm{~kg} / \mathrm{m}^{3}$ ), quantum gravity induces a repulsive pressure so strong not only to stop the collapse, as in non-singular black holes, but to make the collapsing matter 'bounce back', thus triggering an explosion and the end of the black hole phase. Indications for this very exotic scenario come from loop quantum cosmology, an application of loop quantum gravity to simple cosmological models. In that context, extensive model building shows that using the dynamics of loop quantum cosmology, the initial Big Bang singularity is replaced by a 'Big Bounce': the universe does not come from an initial singularity, but from a previously contracting phase bouncing back once the critical Planck density is reached [12]. Rovelli's scenario extends this possibility from cosmology to gravitational collapse. From the outside, the exploding black hole phase triggered by quantum gravity looks like a white hole metric, namely the time reverse of a black hole, in which all matter is expelled from the white horizon; for this reason, this scenario is often called 'black hole to white hole transition'. Notice that this process is classically impossible: the two trajectories of matter (the collapsing and the exploding phases), or equivalently the two metrics (black hole evolving into a white hole) are not connected by any classical solution of Einstein's equations. The scenario is thus based on the well known quantum mechanical tunnelling effect: two classical configurations not connected by any trajectory could nonetheless have a non zero possibility of being joined by a tunnelling. The most famous example is the radioactive decay of an unstable isotope: 


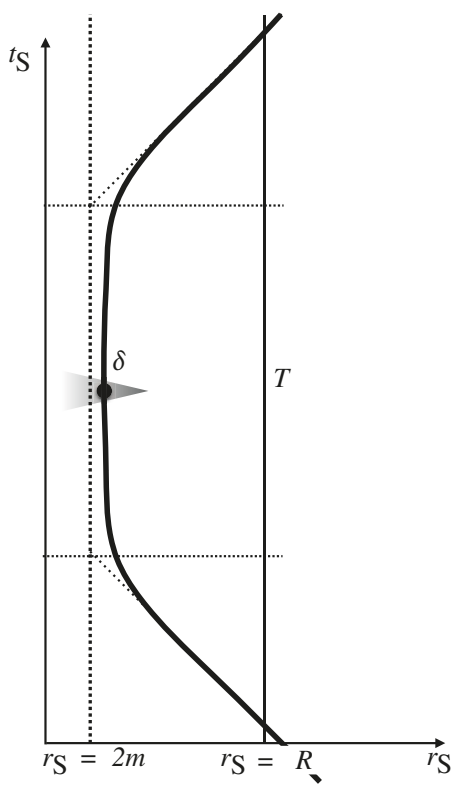

Figure 3

a quantum particle confined to the nucleus can have a non-zero probability of escaping its potential barrier. The key question is to estimate the probability of the tunnelling, hence, the lifetime of the isotope.

Like in all relativistic settings, the notion of time depends on the observer. To understand what is the relevant lifetime discussed here, let us consider the above tunnelling picture from the viewpoint of an observer outside the black hole. From his perspective, he can not see the matter falling in the event horizon and reaching Planckian energy density. Hence, what he would see is the matter falling towards the event horizon, increasingly slowing down and being redshifted as in the classical collapse, until at some point the tunnelling occurs, and the bouncing matter comes out again in the now white hole metric. The complete trajectory of the matter is reproduced in the figure 3 .

Notice that the outside observer concludes that non-perturbative quantum effects causing the bounce must happen at the horizon scale, point $\delta$ in the picture. Here $t_{S}$ and $r_{S}$ are the radial and temporal coordinates of the external observer, and $T$ is the observed lifetime of the black hole.

It is this lifetime as measured from the outside that is crucial for the exploding black hole picture to make sense. In particular, the half-life could be shorter than the extremely long time it takes for Hawking's radiation to make the black hole evaporate. The tunnelling and associated explosion would then occur before complete evaporation. In other words, it is a non-perturbative phenomenon, that could not be predicted in Hawking's perturbative 
scheme. Notice that in this picture Hawking radiation plays a sub-leading role, much like a dissipative effect. It is there and affects the evolution of the black hole, but it is not the principal cause of the black hole's finite life. This scenario shares with non-singular black holes the key point of a regular centre, with no singularity, and associated later purification of the Hawking radiation once the event horizon has disappeared. However, while in the non-singular black hole picture it is the Hawking radiation that makes the horizon evaporate completely, in this alternative picture it is the non-perturbative quantum tunnelling that intervenes before and makes the black hole explode. The other crucial difference between the two scenarios is that in the non-singular black hole, strong quantum gravity effects are only invoked near the centre of the black hole. In the exploding black hole instead, the outside observer sees strong quantum gravity effects at the horizon scale.

The idea can be tested in principle with any approach to quantum gravity that is sufficiently developed to tackle this issue. Loop quantum gravity has reached this level, and in the past year we have worked in a collaboration led by Rovelli [II] to set up the ground for the calculation. To understand how this 'bounce' could be possible, it is necessary to know some simple details about loop quantum gravity. In this description of quantum gravity, the very fabric of spacetime is made by a collection of 'atoms of space', much like my desk is made out of atoms. Such atoms of space would have the dizzyingly small size of $10^{-35} \mathrm{~m}$, and would thus be impossible to see at large, macroscopic scales: their collective behaviour is indistinguishable from the continuous, smooth spacetime of general relativity and quantum field theory. Only when the matter density reaches Planckian values, it is possible to start interacting with the individual atoms of space. The calculation we have set up shows that at Planckian energy densities, there is a transfer of energy from the in-falling matter to the atoms of space. The latter, previously quietly sitting in a coherent state describing a smooth black hole geometry, absorbs enough energy to tunnel into a new configuration, where they settle down into a coherent state describing the white hole. Energy is exchanged back to the matter, and the classical evolution under the white hole metric is restored. The tricky point is to compute the actual probability amplitude for this phenomenon, and because of the complexity of our formula, we still do not know the answer. Nonetheless, we can already advance a few interpretations of the outcome.

Assuming for simplicity spherical symmetry and time-reversal symmetry, the lifetime can depend only on the mass $M$ of the black hole, and can be a priori any function. However, there are strict bounds that can be derived from theory and experiments. Lifetimes proportional to $M$ or $M \log M$ for instance, are incompatible with observations: astrophysical black holes of solar masses would undergo tunnelling in a matter of seconds, contrarily to their observed stability. If the loop quantum gravity calculation predicts a lifetime that is too short, the mechanism can be ruled out. One the other hand, lifetimes proportional to $M^{3}$ or longer would mean that the black hole would evaporate under Hawking radiation before tunnelling, making the phenomenon not relevant, and switching interest back to non-singular black holes. Hence, the only interesting outcomes would be lifetimes of order $M^{2}$. Reintroducing physical units, this means

$$
\tau \sim \sqrt{\frac{G^{3}}{\hbar c^{7}}} M^{2}
$$


Notice that the effect depends on the inverse square root of $\hbar$, which indicates that this cannot be a perturbative phenomenon, as expected.

The most interesting aspect of this possibility is that it opens a new window for quantum gravity phenomenology: If the black hole tunnelling hypothesis is true, black holes would actually be exploding, and such explosions could well be observable in telescopes! The reader should appreciate the fact that quantum gravity effects are expected to be so tiny to be virtually invisible. For instance, we know that Newton's law is modified at small scales by graviton loops, much like Coulomb's law. However, while the modifications to Coulomb's law occur at scales of order $10^{-15} \mathrm{~m}$, and have been abundantly tested to high precision, the modifications to Newton's law occur at the ridiculously small scales of $10^{-35} \mathrm{~m}$, to be compared with the smallest scales accessible today, $10^{-18} \mathrm{~m}$. The difference, $10^{-17} \mathrm{~m}$, is like saying that we are trying to identify the position of Rome using a ruler the size of the galaxy! Most tests lie similarly well outside the experimental domain, thus making quantum gravity typically a purely theoretical and mathematical quest. On the other hand, a lifetime proportional to $M^{2}$ implies that primordial black holes of lunar-size mass could be exploding today and yield observable signals [8]. A component of the expected resulting signal is tantalisingly similar to the recently observed Fast Radio Bursts [13], and this intriguing possibility has been presented also to the general public [14]. Tunnelling of black holes could therefore be a testable quantum gravity prediction, and we are looking forward into developing it forward in future research.

\section{References}

[I] https://en.wikipedia.org/wiki/Cygnus_X-I

[2] Black-hole analogue works like a laser, Physics World, Oct 15, 2014 [http://physicsworld. com/cws/article/news/20l4/oct//5/black-hole-analogue-works-like-a-laser]

[3] C. Rovelli, Quantum gravity, Cambridge University Press 2004

[4] S. A. Hayward, Formation and evaporation of regular black holes, Phys. Rev. Lett. 96 (2006) 31103 [gr-qc/0506/26].

[5] V. P. Frolov, Information loss problem and a 'black hole' model with a closed apparent horizon, arXiv (2014) [1402.5446].

[6] H. M. Haggard and C. Rovelli, "Quantum-gravity effects outside the horizon spark black to white hole tunneling," Phys. Rev. D 92, n. 10, 104020 (20 I5) doi:I 0.I I03/PhysRevD.92.104020 [arXiv: I407.0989 [gr-qc]].

[7] C. Rovelli and F. Vidotto, "Planck stars," Int. J. Mod. Phys. D 23 (2014) no.I2, 1442026 doi:10.1/42/S021827/814420267 [arXiv: 1401.6562 [gr-qc]].

[8] A. Barrau and C. Rovelli, "Planck star phenomenology," Phys. Lett. B 739, 405 (2014) [arXiv: 1404.5821 [gr-qc]].

[9] T. De Lorenzo, C. Pacilio, C. Rovelli and S. Speziale, Gen. Rel. Grav. 47 (2015) n.4, 4l [arXiv: 1412.6015 [gr-qc]]. 
[10] T. De Lorenzo, A. Giusti and S. Speziale, "Non-singular rotating black hole with a time delay in the center," Gen. Rel. Grav. 48 (2016) n.3, 3I [arXiv:I5I0.08828 [gr-qc]].

[II] M. Christodoulou, C. Rovelli, S. Speziale and I. Vilensky, "Realistic Observable in BackgroundFree Quantum Gravity: the Planck-Star Tunnelling-Time," Phys.Rev. D 94 (2016) n.8, 084035 [arXiv: 1605.05268 [gr-qc]].

[12] Big Bang et gravitation quantique, Pour la science, avril-juin 2014 [http://www.pourlascience.fr/ewb/pages/a/article-big-bang-et-gravitation-quantique-32826.php]

[13] Keane E. et al. Nature 530, 453-456 (25 February 2016).

[14] Les sursauts radio rapides: des explosions d'etoiles de Planck?, Futura sciences 2015, janvier 2015 [http://www.futura-sciences.com/sciences/actualites/ trou-noir-sursauts-radio-rapides-explosions-etoiles-planck-56875] 
